\begin{tabular}{cc}
\hline International Journal of Medicine, $9(2)(2021) 63-66$ \\
SPC & Website: $w w w . s c i e n c e p u b c o . c o m / i n d e x . p h p / I J M$ \\
Research paper
\end{tabular}

\title{
Assessment of laboratory test request forms for completeness
}

\author{
Chioma Udeh ${ }^{1}$, Olatunde Olayanju ${ }^{1,2, *, \#, N n a e m e k a ~ A w a h ~}{ }^{1}$, Olabisi Bamidele ${ }^{2}$, Bola \\ Eseile $^{1}$, Onyinye Okonkwo ${ }^{3}$, Gabriel Odok ${ }^{1}$ \\ ${ }^{1}$ Chemical Pathology Department, University College Hospital, Ibadan \\ ${ }^{2}$ Chemical Pathology Department, Babcock University Teaching Hospital, Ilisan \\ ${ }^{3}$ Anatomic Pathology Department, University College Hospital, Ibadan \\ "Joint first author \\ *Corresponding author E-mail: olayanjuo@babcock.edu.ng
}

\begin{abstract}
Background: Laboratory test request forms usually accompany patient's samples to the laboratory, providing biodata and clinical details of the patient. This information is for purposes of identification and guiding pathologists to accurately interpret patient's result. Clinicians however do not usually provide all the required information, thus making interpretation difficult. The frequency of such incompleteness is assessed in this study.

Methods: Laboratory request forms received at the Chemical Pathology laboratory between July and September 2020 were assessed for completeness of all the required parameters. Parameters analyzed in this study included age, gender, hospital number, location, clinical information, name of requesting physician and the date of request. Frequency of missing parameters were expressed as proportions (\%) of the total omissions.

Results: There were 1906 request forms received during the course of this study and 789 (41.4\%) of them had at least one missing parameter. Apart from patients' names, nature of sample and the requested investigation, all other parameters were omitted at one time or the other making a total of 1117 omissions. Age $(287 ; 25.7 \%)$ hospital number $(264 ; 23.6 \%)$ and clinical information $(246 ; 22 \%)$ were the most commonly omitted parameters. Majority of the omissions (69\%) were from the outpatient clinics, $20 \%$ were from the wards while $10 \%$ of the forms had no ward or clinic indicated.

Conclusion: Incomplete filling of laboratory test request forms is a regular occurrence among clinicians. Effort must be made to continually sensitize them of the importance of each of the required parameters to ensure a visible improvement.
\end{abstract}

Keywords: Assessment; Clinical Information; Incompleteness; Laboratory; Request Forms.

\section{Introduction}

Clinical laboratory places a huge emphasis on quality management to ensure that accurate and reliable results are produced to facilitate patients' care. [1], [2] All of the preanalytical, analytical and postanalytical phases of clinical laboratory practice are not entirely foolproof, thus, they are thoroughly monitored to minimize potential errors. [3], [4] The introduction of quality control in the analytical processing of specimen, which is mostly instrument-driven, has virtually eliminated laboratory errors thereof, compared to the preanalytical and the post analytical phases which are essentially human-driven.[5] It is widely known that about $70 \%$ of all laboratory errors occur during the preanalytical phase, and one of the common culprits is the improper filling of laboratory test request forms. [4], [6] In Africa, especially in resource poor settings where laboratory information system is a luxury, handwritten request forms are a common practice, and researches have shown that this practice is prone to errors resulting from illegible handwriting, use of nonstandard abbreviations and incomplete entry of necessary data on the request forms. [7], [8]

Request forms are designed to obtain vital biodata and clinical information from laboratory clients whose sample is being analyzed. [9] When such information is absent or incomplete, they not only constitute a bottleneck in communication between the laboratory and the ward, they also lead to difficulty with interpreting the results and consequently undermine patients' management. [10], [11] It also hinders other postanalytical laboratory services such as interpretative commenting and reflective testing, which have proven to be beneficial and provided additional support to clinician in optimizing patients' management. [12] Furthermore, laboratory test request forms with incomplete information constitute a major difficulty in generating data for research and administrative purposes including audit, reviews, aposteriori reference intervals derivation, amongst several others. Incomplete information on test request forms are common in large hospitals due to the large patient turn out and the busy nature of the services in most of their clinics, wards and emergency rooms. [13] This study was designed to determine the frequency of these omissions with the aim of sensitizing clinicians and propelling them to initiate measures aimed at visible improvement over time. 


\section{Materials and methods}

This was a cross-sectional study conducted at the Chemical Pathology laboratory of the University College Hospital, Ibadan, a tertiary hospital located in the Southwest region of Nigeria. Consecutive request forms received from the wards, clinics and other sections of the hospital between July and September 2020 were examined in this study. Parameters that were examined for complete entry were patients' biodata, data regarding clinical information and the requesting team and the source of the request. Data were obtained directly from the request forms and recorded in a database from which the final analysis was done.

Patients' biodata examined were age and gender, clinical information included clinical diagnosis, hospital number and physician in charge. Other information included wards, clinics and the date samples were sent. The presence or absence of these parameters were recorded in the database and frequency of occurrence from each of the wards were determined. Data analysis was done using Statistical Package for Social Sciences SPSS version 27 (IBM Inc., Armonc, NY).

\section{Result}

A total of 1906 request forms were received in the chemical pathology laboratory within the study period and while 789 (41.4\%) of them had at least one missing parameter, $183(9.6 \%)$ had multiple missing parameters. All the parameters examined under patients' biodata and clinical information had varying degrees of incompleteness making up to 1117 missing parameters in total. The most commonly omitted parameter was patients' age, which was missing in about one quarter $(26 \%)$ of all form examined, closely followed by hospital number (24\%) and clinical information (22\%). The frequency of other missing parameters is reported in Table 1. Name of patient, nature of specimen and required investigation were present in all the request forms reviewed.

Table 1: Frequency of Missing Parameters in Test Request Forms

\begin{tabular}{lll}
\hline Missing Parameter & Number $(\mathrm{N}=1117)$ & Percentage \\
\hline Age & 287 & 25.7 \\
Gender & 162 & 14.5 \\
Clinical information & 246 & 22.0 \\
Patients' location (ward/ clinic) & 121 & 10.8 \\
Name of requesting physician & 132 \\
Hospital Number & 264 \\
Date of request & 26 \\
\hline
\end{tabular}

Request forms without any indication of which ward or clinic they emanated from were 121, making $10.8 \%$ of all incompleteness. In those on which patient's location were indicated, majority of the missing parameters $(69.4 \%)$ were from the outpatient's clinics with the highest number of occurrences coming from the medical outpatient (MOP; 19.1\%), staff clinic (16.4\%), general outpatient (11.6\%) and the surgical outpatient $(10.3 \%)$ clinics in that order. Though, the inpatient ward contributed only about $20 \%$ to the total number of missing parameters, almost all the wards in the hospital were involved with the emergency department (ED) topping the list during the study period (3.6\%); Table 2 .

Table 2: Frequency of Parameters Missing from Different Hospital Sources

\begin{tabular}{lll}
\hline Ward & Number $(\mathrm{N}=1117)$ & Percentage \\
\hline Outpatient clinic $(\mathrm{n}=775 ; 69.4 \%)$ & 129 & 11.6 \\
GOPD & 183 & 16.4 \\
Staff Clinic & 10 & 0.9 \\
CHOP & 213 & 19.1 \\
MOP & 115 & 10.3 \\
SOP & 59 & 5.3 \\
GYNAE & 18 & 1.6 \\
EYE & 7 & 0.6 \\
ANC & 41 & 3.7 \\
CTAG & 221 & 19.8 \\
Inpatient wards & 121 & 10.8 \\
Noward & & \\
\hline
\end{tabular}

GOPD $=$ General outpatient department clinic, $\mathrm{CHOP}=$ Children outpatient clinic, $\mathrm{MOP}=\mathrm{Medical}$ outpatient, $\mathrm{SOP}=\mathrm{Surgical}$ outpatient, $\mathrm{GYNAE}=\mathrm{Gynae}-$ cology clinic, $\mathrm{ANC}=$ Ante-natal clinic, $\mathrm{CTAG}=$ Chief Tony Anenih geriatric clinic

\section{Discussion}

Laboratory test request form is a medium of communication between physicians managing patients and the laboratory where the tests are carried out, especially in settings where electronically generated requests are not available. [14] It is an essential tool for a patient-centered communication skill which all clinician must embrace adequately. [15] Data entry on the request forms, essentially hand-written, provides a substantial information about the patients to the pathologists in the laboratory; this helps to improve the interpretative comments they provide to guide the physicians in making or confirming patients' diagnosis and instituting appropriate treatment. [16] These data entry is often incomplete thereby weakening the quality of support the pathologists provide for the clinicians and sometimes compromising patients' care. A little over $40 \%$ of forms analysed in our study had at least one of such omissions or the other, and both outpatient clinics and inpatients wards were involved. These findings are corroborated by several other local and international studies. [7], [17-20] Apart from the patients' names, tests requested and nature of samples which were present on all the forms examined, almost all the other major parameters were frequently omitted at one time or the other in the course of the study, a reflection of what is regularly obtainable. This is similar to what other studies have reported, and it is understandably so.[19], [20] The patient's name is a major identifier, and it appears to be reflexly written on the form once the test is indicated and automatically followed by the nature of the sample no matter how busy the requesting physicians are. It is not clear why the other parameters do not appear to get as much significance. For example, about $11 \%$ of the forms we examined did not have ward or clinic of origin indicated on them, making it highly difficult to communicate with the requesting physicians in situations of dire emergencies when results are flagged. [11] 
In approximately $10 \%$ of all the forms we examined, there were multiple omissions of essential parameters on the request forms, further underscoring how common this practice is. Given the statutory relevance of these parameters to interpretative comments and reflective testing, [21] the omissions may be suggestive of how much importance requesting clinicians accord these essential laboratory services, rather than being a mere recurring oversight. [16] Sometimes, the busy nature of most clinics where attending clinicians are usually overworked may be responsible for these omissions as they pace through consultations in order to attend to the large number of patients waiting to be seen; this is a common occurrence in most resource-poor settings in Africa. [22], [23]

We found that important information including hospital number, which is crucial for patient's identification, especially in database entry and for purpose of patient's confidentiality were missing in a considerable number of the forms. Furthermore, patient's age and clinical information which are critical factors for consideration in interpretative comments, as recommended by the Royal College of Pathologist (UK) guidelines, [24] constitute some of the most commonly omitted parameters. This usually leads to interpretative dilemma for the pathologists especially in cases where age related reference intervals and peculiar medical conditions are involved. [16] In addition, clinical information provides a guide as to whether a patient is newly diagnosed or an old patient, this is helpful in determining quality of treatment based on the result generated; all these cannot be decided on an incompletely filled request form.

Most of the wards and clinics from where request forms emanated had reported cases of incompleteness although they were fewer from the inpatients' wards compared to the outpatient clinics. The common occurrence in big hospitals like our study site is a low doctor-patients ratio where most clinics are crowded with patients waiting to see the few available doctors. [22] The inpatients wards appeared a little better because patients tend to get more attention from doctors, nurses and other health workers thereby reducing the chances of omissions in filling the request forms.

It appears that the problem regarding incompletely filled request forms will linger for a little while, especially in resource-poor settings where laboratory requests are still done manually, in the face of crowded clinics and wards, and limited health personnel. However, in view of the unfavourable implications it has on optimal delivery of services from the laboratory to the clinicians, turn-around time and ultimately patient care, effort must be intensified to gradually reduce the occurrence. [25] Training of laboratory receptionists who are the first point of contact with the forms, to identify poorly completed forms is highly recommended as this may help to reduce the number at the initial phase. Regular joint meetings between the laboratory and the clinicians, where these issues are discussed and re-emphasized and a gradual integration of laboratory information system into clinical laboratory practice will substantially improve on the frequency of the omissions. [26], [27]

\section{Conclusion}

Our study showed that incomplete filling of test request forms is a very common occurrence among clinicians. Other than patient's name, test indicated and the nature of sample, parameters indicated for filling on request forms were usually ignored for reasons that are not clear; interrogating clinicians in a well-coordinated study may provide a clue. Efforts should be intensified at continuously sensitizing clinicians about the significance of providing all the required information, and also empowering laboratory receptionists to identify such forms at entry points. Though this study is limited by the singularity of the department of study, we are inclined to believe that the same set of clinicians involved would likely demonstrate similar attitude to other laboratories in the hospital

\section{Conflict of interest}

Authors have nothing to declare

\section{References}

[1] Lippi G, Becan-McBride K, Behulova D, Bowen RA, Church S, Delanghe J. Pre-analytical quality improvement: in quality we trust. Clin Chem Lab Med 2013; 51(1): 229- 41. https://doi.org/10.1515/cclm-2012-0597.

[2] Barak M, Jaschek R. A new and effective way of preventing preanalytical laboratory errors. Clin Chem Lab Med 2013; 50(4): 1-4.

[3] Njoroge SW, Nichols JH. Risk management in Clinical Laboratory. Ann Lab Med 2014; 34(4): 274- 8. https://doi.org/10.3343/alm.2014.34.4.274.

[4] Teshome M, Worede A, Asmelash D. Total Clinical Chemistry Laboratory Errors and Evaluation of the Analytical Quality Control Using Sigma Metric for Routine Clinical Chemistry Tests. Journal of Multidisciplinary Healthcare 2021; 14: 125. https://doi.org/10.2147/JMDH.S286679.

[5] Stankovic AK. The laboratory is a key partner in assuring patient safety. Clinics in laboratory medicine 2004; 24(4): 1023-35. https://doi.org/10.1016/j.cll.2004.05.017.

[6] Lippi G, Chance JJ, Church S, et al. Preanalytical quality improvement: from dream to reality. Clin Chem Lab Med 2011; 49: 1113-26. https://doi.org/10.1515/CCLM.2011.600.

[7] Olaniru BO, Alla OJ, Michael E P., et al. Assessment of Patients' Medical Laboratory Request Forms for Compliance in Jos University Teaching Hospital, Jos-Nigeria. Am J Biomed Sci \& Res 2019; 6(4): AJBSR.MS.ID.001056. https://doi.org/10.34297/AJBSR.2019.06.001056.

[8] Schroeder LF, T. A. Medical laboratories in sub- Sahara Africa that meet international quality standards. Am J Clin Pathol 2014; 141(6): 791-5. https://doi.org/10.1309/AJCPQ5KTKAGSSCFN.

[9] WHO. World Health Organisation. Laboratory quality stepwise implementation tool: develop a request form for laboratory testing. www. extranet.who.int/lqsi/content/develop-request-form-laboratory-testing. Accessed on the 7th July 2021.

[10] Wians FH. Clinical laboratory tests: which, why, and what do the results mean? Laboratory Medicine 2009; 40(2): 105-13. https://doi.org/10.1309/LM404L0HHUTWWUDD.

[11] Nutt L, Zemlin AE, T. ER. Incomplete laboratory request forms: the extent and impact on critical results at a tertiary hospital in South Africa. Ann Clin Biochem 2008; 45: 463- 6. https://doi.org/10.1258/acb.2008.007252.

[12] Imoh L, Onyenekwu C, Inaku K, et al. Multicenter Survey of Physicians' Perception of Interpretative Commenting and Reflective Testing in Nigeria. EJIFCC 2021; 32: 85-97.

[13] Adamu S, Mohammed A, El-Bashir JM, Abubakar J, Mshelia D. Incomplete patient data on chemical pathology laboratory forms in a Tertiary Hospital in Nigeria. Annals of Tropical Pathology 2018; 9(1): 47. https://doi.org/10.4103/atp.atp 44 17.

[14] Adu M. The lab requisition form: A vital tool for quality patient care. Annals of Biomedical Sciences 2020; 19(2): 105-8.

[15] Levinson W, Lesser CS, Epstein RM. Developing physician communication skills for patient-centered care. Health Affairs 2010; 29(7): 1310- 8. https://doi.org/10.1377/hlthaff.2009.0450.

[16] Vasikaran S. Interpretative commenting. The Clinical Biochemist Reviews 2008; 29(Suppl 1): S99.

[17] Jegede F, Abdulrahman SA, Mbah HA, Dakata A, Gwarzo DH, Kuliya-Gwarzo A. Evaluating laboratory request forms submitted to haematology and blood transfusion departments at a hospital in Northwest Nigeria. African journal of laboratory medicine 2016; 5(1): 1-6. https://doi.org/10.4102/ajlm.v5i1.381. 
[18] Kipkulei JC, Lotodo TC. Evaluation of the completeness in the filling of laboratory request forms submitted to the haematology laboratory at a Tertiary Hospital in Kenya. Health 2019; 11(7): 862-8. https://doi.org/10.4236/health.2019.117069.

[19] Adegoke OA, Idowu AA, Jeje OA. Incomplete laboratory request forms as a contributory factor to preanalytical errors in a Nigerian teaching hospital. African Journal of Biochemistry Research 2011; 5(3): 82-5.

[20] Alagoa PJ, Udoye EP. Laboratory Request Forms- How Well do Doctors Fill Them? A Look at the Practice at the Niger Delta University Teaching Hospital, Okolobiri, Bayelsa State, Nigeria. Nigerian Health Journal 2015; 15(1): 14-7.

[21] Verboeket-van de Venne WP, Aakre KM, Watine J, Oosterhuis WP. Reflective testing: adding value to laboratory testing. Clinical chemistry and laboratory medicine 2012; 50(7): 1249-52. https://doi.org/10.1515/cclm-2011-0611.

[22] Bank TW. Physicians per 1000 people - sub-Sahara Africa. World Health Organization's Global Health Workforce Statistics, OECD, supplemented by country data.http:// data.worldbank.org/indicator/SH.MED.PHYS.ZS?locations=ZG. Accessed 7th July 2021.

[23] Naicker S, Plange-Rhule J, Tutt RC, Eastwood JB. Shortage of healthcare workers in developing countries--Africa. Ethnicity \& disease 2009; 19(1): 60 .

[24] Marshall WJ, Challand GS. Provision of interpretative comments on biochemical report forms. Annals of clinical biochemistry 2000; 37(6): 758-63. https://doi.org/10.1258/0004563001900066.

[25] Plebani M. Laboratory errors: How to improve pre-and post-analytical phases? Biochemia Medica 2007; 17(1): 5-9. https://doi.org/10.11613/BM.2007.001.

[26] Jastania R. How laboratory information system improves patient safety. Am J Lab Med 2019; 4: 97-100. https://doi.org/10.11648/j.ajlm.20190406.12.

[27] Balas EA. Information systems can prevent errors and improve quality. BMJ Group BMA House, Tavistock Square, London, WC1H 9JR; 2001. 